\title{
Premonitory urges and sensorimotor processing in Tourette syndrome
}

\author{
Sangeerthana Rajagopal ${ }^{\mathrm{a}}$, Stefano Seri ${ }^{\mathrm{b}}$ and Andrea Eugenio Cavanna ${ }^{\mathrm{a}, \mathrm{c}, *}$ \\ ${ }^{a}$ The Michael Trimble Neuropsychiatry Research Group, Department of Neuropsychiatry, BSMHFT and University \\ of Birmingham, Birmingham, UK \\ ${ }^{\mathrm{b}}$ School of Life and Health Sciences, Aston Brain Centre, Aston University, Birmingham, UK \\ ${ }^{\mathrm{c}}$ Sobell Department of Motor Neuroscience and Movement Disorders, Institute of Neurology and University \\ College London, London, UK
}

\begin{abstract}
Most patients with Tourette syndrome report characteristic sensory experiences (premonitory urges) associated with the expression of tic symptoms. Despite the central role of these experiences to the clinical phenomenology of Tourette syndrome, little is known about their underlying brain processes. In the present article we present the results of a systematic literature review of the published studies addressing the pathophysiological mechanisms of premonitory urges. We identified some preliminary evidence for specific alterations in sensorimotor processing at both cortical and subcortical levels. A better insight into the brain correlates of premonitory urges could lead to the identification of new targets to treat the sensory initiators of tics in patients with Tourette syndrome.
\end{abstract}

Keywords: Tourette syndrome, tics, premonitory urges, sensori-motor processing, pathophysiology

\section{Introduction}

Tourette syndrome (TS) is a highly prevalent neuropsychiatric disorder, estimated around $1 \%$ in schoolage children [1]. According to DSM-IV criteria, the onset of tics is before 18 years of age, typically $3-$ 8 years for motor tics and 11 years for phonic tics [2, 3], lasting at least a year. Multiple motor and vocal tics vary in localisation over time and peak in severity at around 10-12 years [4-6]. Interestingly, although first described and popularised by Georges Gilles de la Tourette, an earlier publication by Armand Trousseau seems to offer a description which is more representative of our modern day TS construct with minimal sampling bias [7]. In clinical practice the distinction between tics and habits or repetitive behaviours can be challenging, as all are characterised by various levels of awareness and volitional control [8].

*Corresponding author: Prof. Andrea Eugenio Cavanna, MD $\mathrm{PhD}$, Department of Neuropsychiatry, The Barberry National Centre for Mental Health, Birmingham B15 2FG, UK. Tel.: +44 121 3012317; Fax: +44 121 3012291; E-mail: a.cavanna@ion.ucl.ac.uk.
Premonitory urges (PUs) are not part of the DSM-IV criteria for TS despite the vast majority of patients (77\% of patients over 13 years) experience these symptoms [9-12]. PUs, also called sensory tics, are described as focal or generalised intrusive feelings or sensations driving the individual to seek relief through performance of movements (motor tics) or vocalisations (vocal tics) [13-16]. A study by Leckman et al. [17] found 10 years to be the mean age of PUs awareness, averaging 3 years after tic onset. The awareness latency is suggested to reflect a transition of the processing of sensory information to conscious awareness $[18,19]$. Studies with the Premonitory Urge for Tics Scale (PUTS), a psychometric tool specifically developed to measure the severity of PUs [20,21], have highlighted that awareness of PUs can be a maturational process, independent of tic onset. The most frequently reported localisations of PUs are the palms, shoulders and throat, although $40 \%$ of patients localise them exclusively in the muscle, and the remainders in their joints or skin. The percentage of patients with TS or other chronic tic disorders experiencing PUs varies depending on how the urge is defined [21-23]. 
It has been proposed to categorise PUs as sensory (SUs), cognitive (CUs) or autonomic urges (AUs) [24]. SUs are focal or generalised muscular-skeletal or visceral-sensations, whereas CUs are feelings of incompleteness or 'just-right' perceptions and AUs overlap with symptoms of autonomic dysfunction, such as sweating, palpitations and nausea. Interestingly, urges can also be bound to external stimuli e.g. in automutilatory tics where specific angulation of objects can trigger tics, which are characteristically suggestible by both audio and visual cues [3]. The exact role and significance of PUs is at present unknown. It has been postulated that they could reflect subjective experiences of neural dysfunction below tic-production threshold or heightened attention to physical sensations [8]. PUs may be an illumination of fragments of innate behaviour, closely involved in the orchestration of behavioural programmes. Central to this idea is the binding role of the basal ganglia to allow movement execution in sight of convergent information from the functionally distinct cortico-subcortical circuits, which can account for the heterogeneity of TS symptoms [25, 26].

Importantly, about $90 \%$ of patients with TS can also present with co-morbid behavioural problems, mainly attention-deficit and hyperactivity disorder (ADHD), obsessive-compulsive disorder (OCD), affective disorders and impulse control disorders [27-29]. With this in mind, clinical subdivisions may be appropriate, from 'pure TS' (simply motor and vocal tics) to 'fullblown TS' (tics plus coprophenomena, echopenomena and/or paliphenomena) and 'TS-plus' (with psychiatric co-morbidities) [30]. PUs have been reported to cause more distress to patients with TS than tics, which appear semi-voluntary in response to inner urges [4, 31-33]. Self-awareness of these subjective symptoms could improve the ability to suppress tics, as shown by the effectiveness of behavioural strategies for tic control such as exposure and response prevention and habit reversal therapy $[17,34,35]$.

Due to their intrinsic subjectivity the neural correlates of PUs are difficult to investigate. However a better understanding of their pathophysiology might have significant clinical implications. In the present article we present the results of a systematic literature review of the published studies addressing the pathophysiological mechanisms of PUs.

\section{Methods}

We conducted a systematic literature review according to the Prisma guidelines [36] using the search terms 'tic*', 'tourett*', 'anxiety', 'mechanism', 'urge*', 'sensor', across the scientific databases EMBASE, HMIC, Medline and PsycINFO. We excluded from the review studies that did not focus on human subjects and all non-English literature. Additional relevant publications were identified by snowballing reviewed papers; "grey" literature was retrieved from Google searches using the above-mentioned search terms.

\section{Results}

Our systematic literature review identified five studies specifically focussing on the pathophysiology of PUs in patients with TS and these are summarised in Table 1. Two articles were neurophysiological studies (focussing on the Bereitschaftspotential and Somatosensory Evoked Potentials respectively), two were neuroimaging studies (structural and functional magnetic resonance imaging) and one was a recent review paper.

\section{Discussion}

\subsection{The pathophysiology of premonitory urges}

Despite their central role in subjective tic phenomenology, PUs have received little attention and few studies have focused on this intriguing phenomenon in patients with TS. The reviewed literature highlights several hypotheses on the pathophysiological bases of PUs. Converging evidence supports the presence of sensory gating dysfunction. Specifically, gating dysfunction in TS may cause excessive inflow of somatosensory information, possibly generating PUs by acting on particular cortico-striatal synapses through LTP and increasing SMA activation. Abnormal dopamine function seems also to be implicated. Dopamine transporter hyperactivity can play a central role, as dopamine can have relevant effects on particular cortico-striatal synaptic strengths. Moreover, the striosomal compartment of the striatum is interconnected with limbic structures and can modulate matrix activity via dopaminergic neurons from the substantia nigra-pars compacta ( $\mathrm{SNpc}$ ), influencing motivation-based behaviour. Of note, there is considerable overlap with the neurobiological bases of reward experiences, which involve the mesolimbic dopaminergic system and prefrontal cortical regions interlinked with the ventral striatum. Anatomically, convergence and topographical organisation of functionally related 


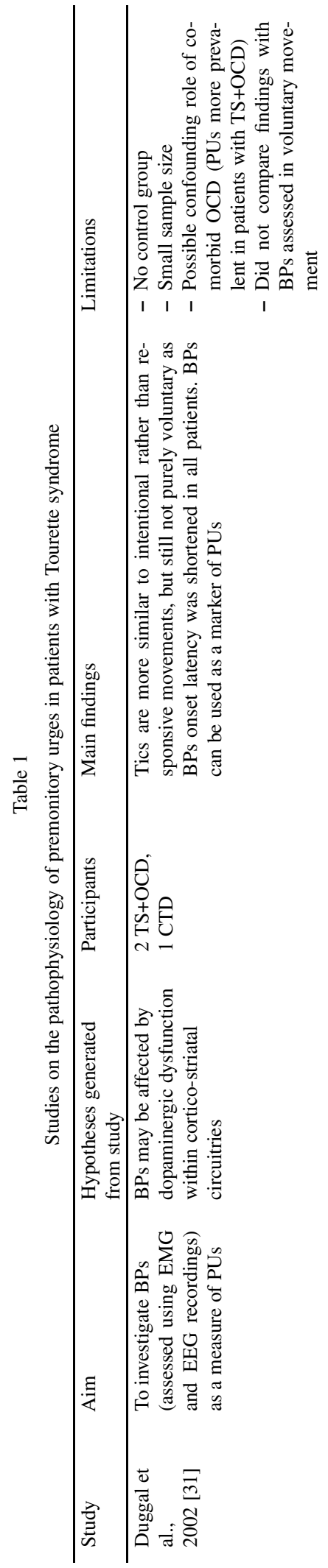

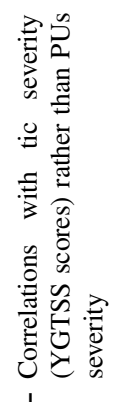

.

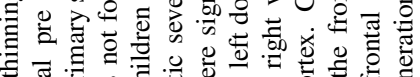

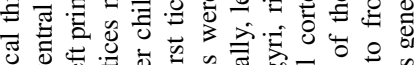

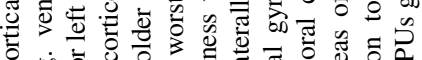

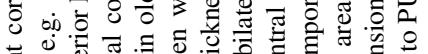

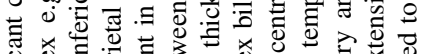

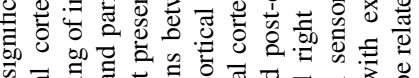

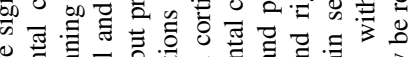

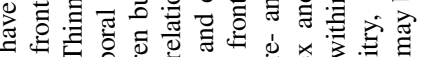

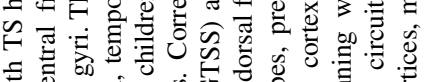

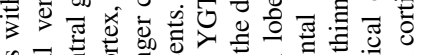

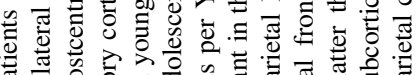

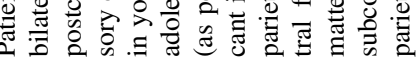

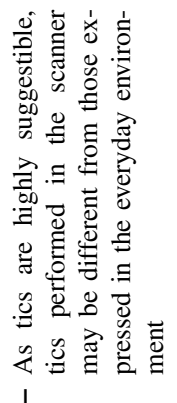

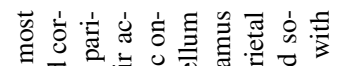

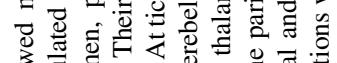

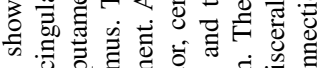

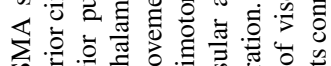
的牙

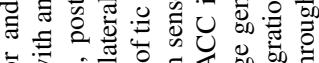

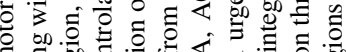

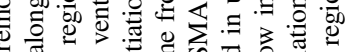

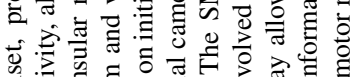

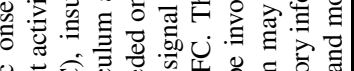

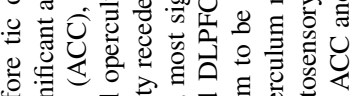

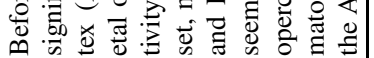

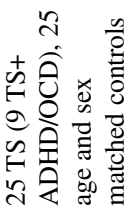

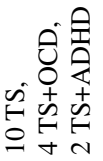

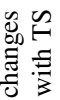

률.

过

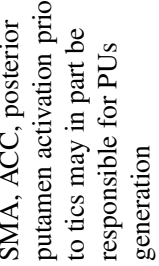

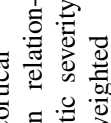

응

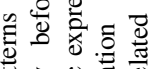

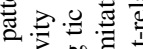

记

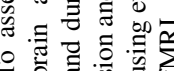

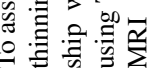

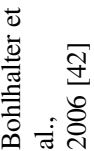




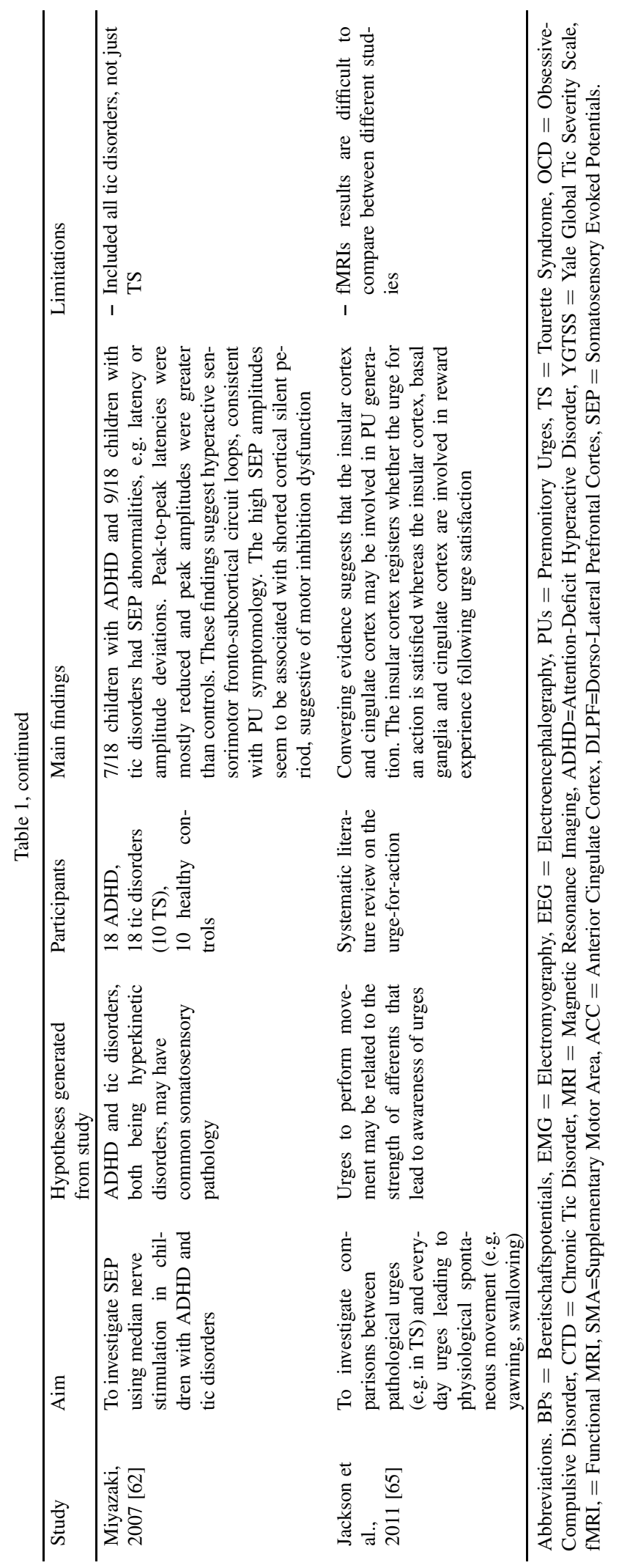


cortico-striatal projections entertain particular striatal regions while impressing functional subdivision of the striatum. The dorsolateral striatum is predominantly 'somatosensory'-related, the intermediate portion 'associative' and the ventromedial 'limbic', which allows these areas to be implicated in different aspects of movement and complex behaviour [37,38].

\subsection{Sensorimotor gating}

The most accredited pathophysiological theory emerging from this review, suggests that PUs are the expression of a 'sensorimotor gating' dysfunction. Sensorimotor gating is responsible for the modulation in the allocation of neural resources in the presence of competing sensory and cognitive information from the multitude present in the environment [39]. When premotor and prefrontal cortices initiate a motor pattern, the striatum may allow initiation if agreeable with activity of other convergent corticostriatal inputs, while suppressing competing motor programmes. Therefore, basal ganglia may be involved in selection of motor and behavioural patterns within appropriate context [40] via this gating mechanism. One study [37] found that externally paced movement created increased activity in the anterior caudate-putamen, a possible pivotal site for this 'gate' mechanism. Additionally, electrical stimulation of the SMA in non-TS individuals produced an urge to move $(n=13)$ or unusual sensations $(n=9)$ [41]. This suggests SMA's involvement in movement intentions, especially as the SMA and the anterior cingulate cortex (ACC) have dense dopaminergic projections from midbrain structures such as the substantia nigra ( $\mathrm{SN}$ ) and its activation prior to tic onset can potentially generate PUs via limbic-motor connections [42]. In relation to these pathways, other results show low metabolic activity of the ventral striatum and limbic regions and increased activity in SMA and sensory association regions [5].

\subsection{Structured event complexes}

The orbitofrontal-cortex (OFC) is typically associated with social behaviour, emotion and reward, and has been proposed to interact with BG in reward learning. Via sensorimotor gating, the BG can favour initiation of particular 'cognitive pattern generators' in the cortex [43]. Through reinforcement by dopamine influencing long-term-potentiation (LTP) [44] of particular synapses, gating can activate rewarding behaviour. The amygdala, coding for stimulus intensity, also appears to be involved. The prefrontal cortex (PFC) appears to have 'structured event complexes' (SECs)/behavioural patterns that are hierarchical in nature. In OCD models, the ACC seems to play an important role for motivation and error processing in calculation of disparities between expected and gained reward [43]. Therefore, motivation to complete a SEC could be processed through communication between reward regions such as the OFC, activation threshold assigned by the BG, error calculation by ACC and emotional importance by limbic areas. However, prevention from performing SECs is punishing and this punishment is only removed once the behaviour is completed. In OCD models, little relief is experienced because of ACC signalling incomplete behaviour, OFC punishment, anxiety generated in limbic structures and the BG reducing thresholds for compensating SECs. This 'feeling of incompletion' of SECs causes an obsession to gain relief from anxiety. However, in OCD, only a small proportion of the full reward is experienced. A similar process may occur to generate PUs in TS: tics may occur because of the reduced inhibitory BG output because of its reduced threshold possibly due to fewer inhibitory-interneurons [45] and low metabolic activity [5]. The cognitive-psychophysiological model of TS [8] suggests that patients with TS have particular cognitive styles governing 'correct' manners to undertake and organise activities - a perfectionist style. This mechanism, together with physiologically heightened sensory awareness, creates tendencies to attempt too much and reduced relaxation, paralleling ADHD [46]. This in turn creates conflicts between what should be undertaken and what is being done, diminishing feelings of achievement [8].

Interestingly, it seems that PUs may not be the only manifestation of fronto-striatal dysfunction in TS. An investigation undertaken by Eddy et al. [47] into executive function and Theory of Mind in TS revealed alterations in these functions generated by the frontal cortex. Patients and controls were assessed for reticence and verbal fluency in various tasks such as evaluating mental states by looking at images of faces, understanding types of humour and other decision processes. On comparison with controls and even with exclusion of co-morbid OCD, performance in TS was reduced, suggesting dissociation between ventromedial prefrontal cortex and the striatum. The results of different studies corroborate these findings $[48,49]$. Moreover, an MRI study by Draganski et al. [50] found adults with TS to have anterior cingulate, ventrolateral prefrontal and orbitofrontal grey matter volume re- 
duction. Reduced grey matter in orbitofrontal region is thought to be related to reduced impulse control and behavioural inhibition, which are known features of TS [30]. Draganski et al. also found increased dorsolateral putamen volume bilaterally, especially in the subgroup of patients with TS+ADHD, and increased thickness in the left primary somatosensory cortex, with a significant correlation to PU severity as measured by the PUTS scores. PU sensory information triggering motor tics could potentially result in increased volume evidenced in the dorsolateral putamen via plastic remodelling through dorsolateral putamen connections to sensorimotor areas allowing 'sensoryguided' movement. Additionally, neuroleptic exposure could also result in striatal volume increases, and increased thickness of somatosensory cortex can be related to increased myelin thickness [51]. This suggests that PUs may impose greater functional demands for integration into motor responses (tics), allowing plasticity-mediated volume change. Alternatively, reduced parietal operculum (a secondary somatosensory region) thickness may cause 'rerouting' of its demands to the somatosensory cortex as a compensatory mechanism.

\subsection{Prepulse inhibition}

Weak stimuli preceding startle stimuli can cause reduction in sensitivity to sensory stimuli, resulting in reduced startle reflex magnitudes. This is termed prepulse inhibition (PPI) and is thought to be linked with basal ganglia's role in sensorimotor gating processes, shaped by genetic, developmental and hormonal factors [39]. Reduced PPI in TS appears to correlate with the presence of interfering cognitive, motor and sensory information [52]. Interestingly, this is not specific to TS, but is also found in other neuropsychiatric conditions, e.g. Huntington's disease and schizophrenia [22]. On prepulse+pulse experiments, mixed D1/D2 agonist apomorphine increased the startle magnitude and reduced PPI, possibly reflecting genetic differences in D1 and D2 receptor sensitivity [39]. Converging evidence suggests that brain structures involved in PPI may incorporate parallel connections involving limbic areas, ventral striatum and pallidum, with the cortical-subcortical projections overlapping the startle circuit at the level of the reticularis pontis caudalis [53]. Lesions of the dorsal striatum (somatosensory component) have also been shown to result in PPI reduction [54]. The PPI model suggests parallels between tics and adapted startle reflexes [55], in line with the learning model of TS [47], where distressing events may create a reflex which later develops into a reinforced tic [56].

\subsection{The dopamine transporter system}

Overactivity of the dopamine transporter system appears to be consistently implicated in PUs generation [57]. A study using amphetamine challenge in 7 patients with TS found that mean putamen intrasynaptic dopamine levels were increased by $21 \%$ [58]. However, PET scans revealed no significant differences between D2-receptors density between TS and control brains. This may be explained by low tonic (basal) DA-levels, presynaptically regulated by D2 and D3-receptors (autoreceptors). Findings of low extrastriatal D2 receptors could also point to tonic/phasic dopamine dysfunction [59]. These findings are consistent with the clinical observations of tic exacerbations triggered by environmental stimuli (e.g. stress and anxiety), leading to increased phasic dopamine release and orbitofrontal and mesolimbocortical dopaminergergic dysfunction. From a developmental perspective, aberrant habits can result from imbalances between dorsolateral-somatosensory, intermediate-associative and ventromedial-limbic striatum. These abnormalities in the prefrontal-ventral-striatal circuitry can in turn cause inappropriate behavioural or motor sequence expression, subjectively experienced as PUs.

\subsection{Striatal tonically active neurons}

Striatal tonically active neurons (TANs) are thought to play a key role in the application of motivational contexts to conditioned sensory-instigated behaviour, executed on anticipation of reward $[57,66]$. These neurons are cholinergic interneurons which are activated by expectation of reward. Interestingly, TANs in the caudate and putamen appear to differ in their encoding of motivational outcomes: they can influence motivation for behaviour, 'go-response' for actions with expected motivational contexts, or both, thus allowing anticipation of rewarding or undesirable circumstances with initiation of 'goal-directed behaviour.' Additionally, TANs are responsive to dopaminergic SNpc inputs, which is a likely participant in reward calculations with inputs from the thalamus [60,64]. Within the context of sensorimotor gating dysfunction in TS, TANs may facilitate formation of abnormal motivational contexts for reward anticipation leading to PUs. Fast-spiking-GABA-interneurons (FSNs) can also be 
implicated, as they receive somatosensory cortex projections. Synchronised oscillatory TAN and FSN firing in conditioned behaviour can modify the activity of their respective striatal projections and favour dopaminergic plasticity of particular striatal synapses possibly involved in PUs generation. According to one hypothesis on the pathophysiology of TS, specific matrix interneurons may dissociate from normal oscillatory rhythm and 'besiege' individuals with sensory experiences $[36,46]$. Both abnormal activity of striatal interneurons $[45,60]$ and hyperpolarisation of particular thalamocortical neurons coupled with irregular GPi firing, may interfere with normal cortical activity and result in PUs as experienced by patients with TS [61]. However, the abnormal cortico-subcortical loop activation may also be of cortical origin, consistent with shortened cortical silent period [60], abnormal sensory-evoked potentials [62] and thinning of premotor and sensorimotor cortical areas $[3,38,63]$.

\subsection{Future perspectives}

Further research is needed to better characterise and understand PUs in patients with TS. Little is known about specific PUs mechanisms underlying different tic subtypes, e.g. whether PUs associated with complex or simple tics or tics in particular anatomical locations have different pathophysiological substrates. It is possible that different neural pathways are identified between different tic subtypes, beyond shared sensorimotor processes. Moreover, studies comparing mechanisms of PUs generation in TS to other disorders such as OCD and schizophrenia may reveal more details about pathophysiological differences, which could also have clinical implications in terms of effectiveness of antipsychotic treatment. Investigations into asymmetry of basal ganglia structural changes in neurodevelopmental disorders such as TS could reveal indications of brain maturation dysfunction. This could in turn have implications for structures involved in PUs generation. Likewise, investigation into whether dysfunctional development of selective cognitive abilities leads to PU awareness may be important. Finally, the possible relationship between PU severity and Bereitschaftspotential also deserves further research, in order to use neurophysiological evidence to better characterise the clinical distinction between voluntary and involuntary actions in response to PUs.

\section{References}

[1] Robertson MM. The prevalence and epidemiology of Gilles de la Tourette syndrome: Part 1: The epidemiological and prevalence studies. J Psychosom Res. 2008: 65; 461-472.

[2] American Psychiatric Association. Diagnostic and Statistical Manual of Mental Disorders - Fourth Edition, Text Revision (DSM-IV-TR). American Psychiatric Press, 2000.

[3] Leckman JF. Phenomenology of tics and natural history of tic disorders. Brain Dev. 2004: 25 Suppl; S24-S28.

[4] Albin RL, Mink JW. Recent advances in Tourette syndrome research. Trends Neurosci. 2006; 29: 175-182.

[5] Braun AR, Stoetter B, Randolph C, Hsiao JK, Vladar K, Gernert J, Carson RE, Herscovitch P, Chase TN. The functional neuroanatomy of Tourette's syndrome: An FDG-PET study. I. Regional changes in cerebral glucose metabolism differentiating patients and controls. Neuropsychopharmacol. 1993: 9; 277-291.

[6] Tourette Syndrome Classification Study Group. Definitions and classification of tic disorders. Arch Neurol. 1993; 50: 1013-1016.

[7] Rickards H, Woolf I, Cavanna AE. Trousseau's disease: A description of Gilles de la Tourette syndrome 12 years before 1885. Mov Disord. 2010; 25: 2285-2289.

[8] O'Connor K. A cognitive-behavioural/psychophysiological model of tic disorders. Behav Res Ther. 2002:40; 113-1142.

[9] Chee KY, Sachdev P. A controlled study of sensory tics in Gilles de la Tourette syndrome and obsessive-compulsive disorder using a structured interview. J Neurol Neurosurg Psychiatry. 1997; 62: 188-192.

[10] Cohen AJ, Leckman JF. Sensory phenomena associated with Gilles de la Tourette's Syndrome. J Clin Psychiatry. 1992; 53: 319-323.

[11] Kwak C, Dat Vuong K, Jankovic J. Premonitory sensory phenomenon in Tourette's syndrome. Mov Disord. 2003; 18 : 1530-1533.

[12] Steinberg T, King R, Apter A. Tourette's Syndrome: A Review from a Developmental Perspective. Isr J Psych Relat Sci. 2010; 47: 105-109

[13] Bliss J, Cohen DJ, Freedman DX. Sensory experiences of Gilles de la Tourette syndrome. Arch Gen Psychiatry. 1980; 37: 1343-1347.

[14] Bullen JG, Hemsley DR. Sensory experiences as a trigger in Gilles de la Tourette's syndrome. J Behav Ther Exp Psychiatry. 1983; 14: 197-201.

[15] Kurlan R, Lichter D, Hewitt D. Sensory tics in Tourette's syndrome. Neurology. 1989; 39: 731-734.

[16] Scahill LD, Leckman JF, Marek KL. Sensory phenomena in Tourette's syndrome. Adv Neurol 1995; 65: 273-280.

[17] Leckman JF, Walker DE, Cohen DJ. Premonitory urges in Tourette's syndrome. Am J Psychiatry. 1993: 150; 98-102.

[18] Banaschewski T, Woerner W, Rothenberger A. Premonitory sensory phenomena and suppressibility of tics in Tourette syndrome: Developmental aspects in youth and adolescents. Dev Med Child Neurology. 2003; 45: 700-703.

[19] Kane MJ. Premonitory urges as "attentional tics" in Tourette's syndrome. J Am Acad Child Adolesc Psychiatry. 1994; 33 : 805-808.

[20] Steinberg T, Schmuel Baruch S, Harush A, Dar R, Woods D, Piacentini J, Apter A. Tic disorders and the premonitory urge. J Neural Transm. 2010; 117: 277-284.

[21] Woods DW, Piacentini J, Himle MB, Chang S. Premonitory Urge for Tics Scale (PUTS): Initial psychometric results and 
examination of the premonitory urge phenomenon in youths with tic disorders. J Dev Behav Pediatr. 2005: 26; 397-403.

[22] Sutherland Owens AN, Miguel EC, Swerdlow NR. Sensory gating scales and premonitory urges in Tourette syndrome. Sci World J. 2011: 11; 736-741.

[23] Prado HS, Rosário MC, Lee J, Hounie AG, Shavitt RG, Miguel EC. Sensory phenomena in obsessive-compulsive disorder and tic disorders: A review of the literature. CNS Spectrums. 2008; 13: 425-432.

[24] Miguel EC, do Rosario-Campos M, Prado H, do Valle R, Rauch SL, Coffey BJ, Baer L, Savage CR, O'Sullivan RL, Jenike MA, Leckman JF. Sensory phenomena in obsessivecompulsive disorder and Tourette's disorder. J Clin Psychiatry. 2000: 61; 150-156.

[25] Peterson BS, Skudlarski P, Anderson AW, Ahang H, Gatnenby JC, Lacadie CM, Leckman JF, Gore JC. A functional magnetic resonance imaging study of tic suppression in Tourette syndrome. Arch Gen Psychiatry. 1998; 55: 326-333.

[26] Tekin S, Cummings J. Frontal-subcortical neuronal circuits and clinical neuropsychiatry: An update. J Psychosom Res. 2002; 25: 647-654.

[27] Freeman RD, Fast DK, Burd L et al. An international perspective on Tourette's syndrome L selected findings from 3,500 individuals in 22 countries. Dev Med Child Neurol. 2000; 42: 436-447.

[28] Robertson MM. Tourette syndrome, associated conditions and the complexities of treatment. Brain. 2000: 123; 425-462.

[29] Rosário MC, Prado HS, Borcato S, Diniz JB, Shavitt RG, Hounie AG, Mathis ME, Mastrorosa RS, Velloso P, Perin EA, Fossaluza V, Pereira CA, Geller D, Leckman J, Miguel E. Validation of the University of São Paulo Sensory Phenomena Scale: initial psychometric properties. CNS Spectrums. 2009; 14: 315-323.

[30] Cavanna AE, Servo S, Monaco F, Robertson MM. The Behavioural Spectrum of Gilles de la Tourette Syndrome. J Neuropsychiatry Clin Neurosci. 2009: 21; 13-23.

[31] Duggal HS, Nizamie SH. Bereitschaftspotential in tic disorders: A preliminary observation. Neurol India. 2002: 50; 487489.

[32] Lang AE. Patient perception of tics and other movement disorders. Neurology. 1991; 41: 223-228.

[33] Moretto G, Schwingenschuh P, Katschnig P, Bhatia KP, Haggard P. Delayed experience of volition in Gilles de la Tourette syndrome. J Neurol Neurosurg Psychiatry. 2011; 82: 13241327.

[34] Himle MB, Woods DW, Conelea CA, Bauer CC, Rice KA. Investigating the effects of tic suppression on premonitory urge ratings in children and adolescents with Tourette's syndrome. Behav Res Ther. 2007; 45: 2964-2976.

[35] Verdellen CWJ, Hoogduin CAL, Kato BS, Keijsers GPJ, Cath DC, Hoijtink HB. Habituation of premonitory sensations during exposure and response prevention treatment in Tourette's syndrome. Behav Modif. 2008; 32: 2215-2227.

[36] Moher D, Liberati A, Tetzlaff J, Altman D, The PRISMA Group. Preferred reporting items for systematic reviews and meta-analyses: The PRISMA Statement. PLoS One. 2009; 6: $1-6$.

[37] Menon V, Glover GH, Pfefferbaum A. Differential activation of dorsal basal ganglia during externally and self paced sequences of arm movements. Neuroreport. 1998: 11;15671573.

[38] Worbe Y, Gerardin E, Hartmann A, Valabréue R, Chupin M, Tremblay L, Vidailhet M, Colliot O, Lehéicy S. Distinct struc- tural changes underpin clinical phenotypes in patients with Gilles de la Tourette syndrome. Brain. 2010: 133; 3649-3660.

[39] Swerdlow, NR, Braff, DL, Geyer, MA. Animal models of deficient sensorimotor gating: What we know, what we think we know, and what we hope to know soon. Behav Pharmacol. 2000: 11; 185-204.

[40] Groenewegen HJ, van den Heuvel, OA, Cath DC, Voorn P, Veltman DJ. Does an imbalance between the dorsal and ventral striatopallidal systems play a role in Tourette's syndrome? A neuronal circuit approach. Brain Dev. 2003: 25 Suppl; S3S14.

41] Fried I, Katz A, McCarthy G, Sass KJ, Williamson P, Spencer SS, Spencer DD. Functional organisation of the human supplementary motor cortex studied by electrical stimulation. J Neurosci. 1991: 11; 3656-3566.

[42] Bohlhalter S, Goldfine A, Matteson S, Garraux G, Hanakawa T, Kansaku K, Wurzman R, Hallett M. Neural correlates of tic generation in Tourette syndrome: An event-related functional MRI study. Brain. 2006: 129; 2029-2037.

[43] Huey ED, Zahn R, Krueger F, Moll J, Kapogiannis D, Wassermann EM, Grafman J. A psychological and neuroanatomical model of obsessive-compulsive disorder. J Neuropsychiatry Clin Neurosci. 2008: 20; 390-480.

[44] Horvitz JC. Stimulus-response and response-outcome learning mechanisms in the striatum. Behav Brain Res. 2009: 199: $129-140$.

[45] Kalanithi PAS, Zheng W, DiFiglia M, Grantz H, Saper CB, Schwartz ML, Leckman JF, Vaccarino FM. Altered parvalbumin-positive neuron distribution in basal ganglia of individuals with Tourette syndrome. Proc Natl Acad Sci USA. 2005: 102; 13307-13312.

[46] Conelea CA, Woods DW. Examining the impact of distraction on tic suppression in children and adolescents with Tourette syndrome. Behav Res Ther. 2008: 46; 1193-1200.

[47] Eddy CM, Mitchell IJ, Beck SR, Cavanna AE, Rickards H. Social reasoning in Tourette syndrome. Cogn Neuropsychiatry. 2011; 16: 326-347.

[48] Eddy CM, Mitchell IJ, Beck SR, Cavanna AE, Rickards H. Altered attribution of intention in Tourette's syndrome. J Neuropsychiatry Clin Neurosci. 2010; 22: 348-351.

[49] Eddy CM, Mitchell IJ, Beck SR, Cavanna AE, Rickards H. Impaired comprehension of non-literal language in Tourette syndrome. Cogn Behav Neurol. 2010; 23: 178-184.

[50] Draganski B, Martino D, Cavanna AE, Hutton C, Orth M, Robertson MM, Critchley HD, Frackowiak RS. Multispectral brain morphometry in Tourette syndrome persisting into adulthood. Brain. 2010; 133: 3661-3675.

[51] Concha L, Livy DJ, Beaulieu C, Wheatley BM, Gross DW. In vivo diffusion tensor imaging and histopathology of the fimbria-fornix in temporal lobe epilepsy. J Neurosci. 2010: 30: $996-1002$.

[52] Castellanos FX, Fine EJ, Kaysen D, Marsh WL, Rapoport JL, Hallett M. Sensorimotor gating in boys with Tourette's syndrome and ADHD: Preliminary results. Biol Psychiatry. 1996: 39; 33-41.

[53] Bakshi VP, Geyer MA. Multiple limbic regions mediate the disruption of prepulse inhibition produced in rats by the noncompetitive NMDA antagonist dizocilpine. J Neurosci. 1998: 20; 8394-8401

[54] Kodsi M, Swerdlow NR. Prepulse inhibition in the rat is regulated by ventral and caudodorsal striato-pallidal circuitry. Behav Neurosci. 1995: 109; 912-928.

[55] Abbruzzese G, Berardelli A. Sensorimotor integration in movement disorders. Mov Disord. 2003: 18; 231-240. 
[56] Verdellen CWJ, Hoogduin CAL, Kato BS, Keijsers GPJ, Cath DC, Hoijtink HB. Habituation of premonitory sensations during exposure and response prevention treatment in Tourette's syndrome. Behav Modif. 2008: 32; 2215-2227.

[57] Kimura M, Yamada H, Matsumoto N. Tonically active neurons in the striatum encode motivational context of action. Brain Dev. 2003: 25 Suppl; S20-S23.

[58] Singer HS, Szymanski S, Giuliano J, Yokoi F, Dogan AS, Brasic JR, Zhou Y, Grace AA, Wong DF. Elevated intrasynaptic dopamine release in Tourette's syndrome measured by PET. Am J Psychiatry. 2002: 159; 1329-1336.

[59] Gilbert DL, Christian BT, Gelfand MJ, Shi B, Mantil J, Sallee FR. Altered mesolimbocortical and thalamic dopamine in Tourette syndrome. Neurology. 2006: 67; 1695-1697.

[60] Peterson B, Riddle MA, Cohen DJ, Katz LD, Smith JC, Hardin MT, Leckman JF. Reduced basal ganglia volumes in Tourette's syndrome using three dimensional reconstruction techniques from magnetic resonance images. Neurology. 1993: 43; 941-949.

[61] Krauthamer G, Dalsass M. Differential synaptic modulation of polysensory neurons of the intralaminar thalamus by me- dial and lateral caudate nucleusand substantia nigra. Brain Res. 1978: 154; 137-143.

[62] Miyazaki M, Fujii E, Saijo T, Mori K, Kagami S. Somatosensory evoked potentials in attention deficit/hyperactivity disorder and tic disorder. Clin Neurophysiol. 2007: 118; 12861290.

[63] Sowell ER, Kan E, Yoshii J, Thompson PM, Bansal R, Xu $\mathrm{D}$, Toga AW, Peterson BS. Thinning of sensorimotor cortices in children with Tourette syndrome. Nat Neurosci. 2008: 11; 637-639.

[64] Schultz W, Tremblay L, Hollerman JR. Changes in behaviourrelated neuronal activity in the striatum during learning. Trends Neurosci. 2003: 26; 321-328.

[65] Jackson SR, Parkinson A, Kim SY, Schuermann M, Eickhoff SB. On the functional anatomy of the urge-for-action. Cogn Neurosci. 2011: 2; 227-257.

[66] Leckman JF, Vaccarino FM, Kalanithi PS, Rothenberger A. Tourette syndrome: A relentless drumbeat driven by misguided brain oscillations. J Child Psychol Psychiatry. 2006: 6; 537-550. 


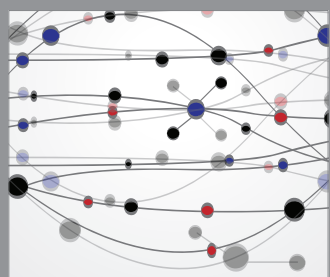

The Scientific World Journal
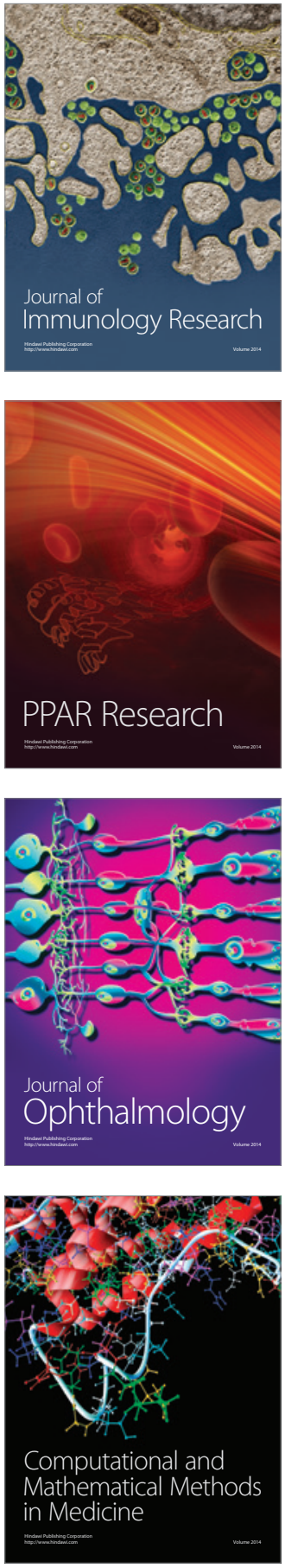

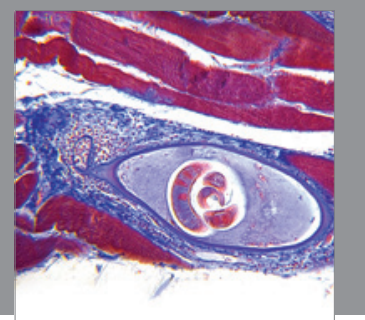

Gastroenterology

Research and Practice
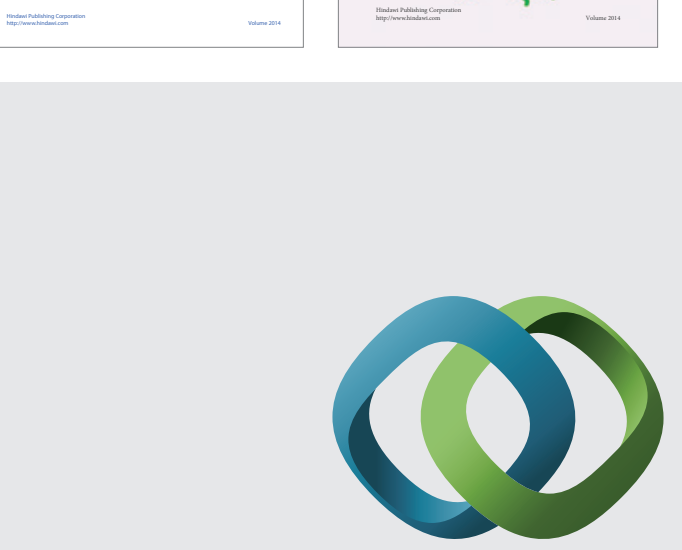

\section{Hindawi}

Submit your manuscripts at

http://www.hindawi.com
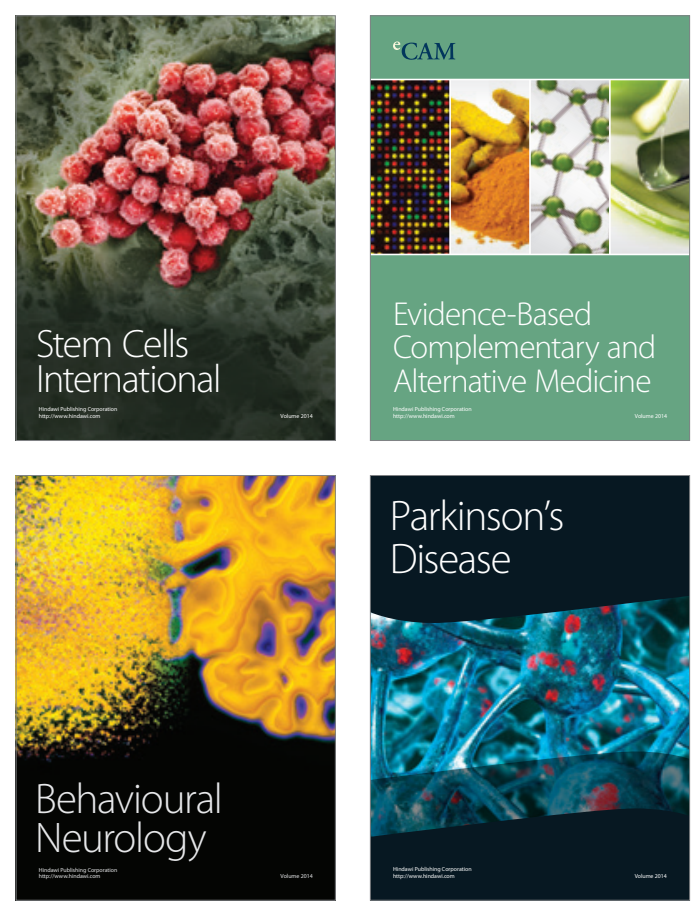

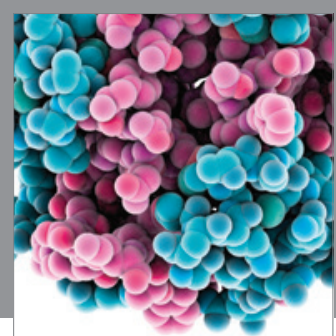

Journal of
Diabetes Research

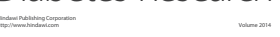

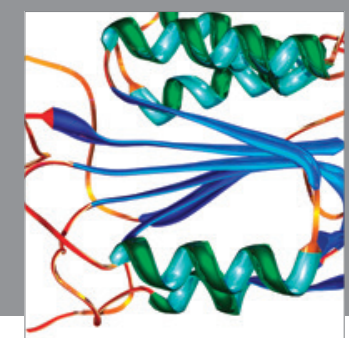

Disease Markers
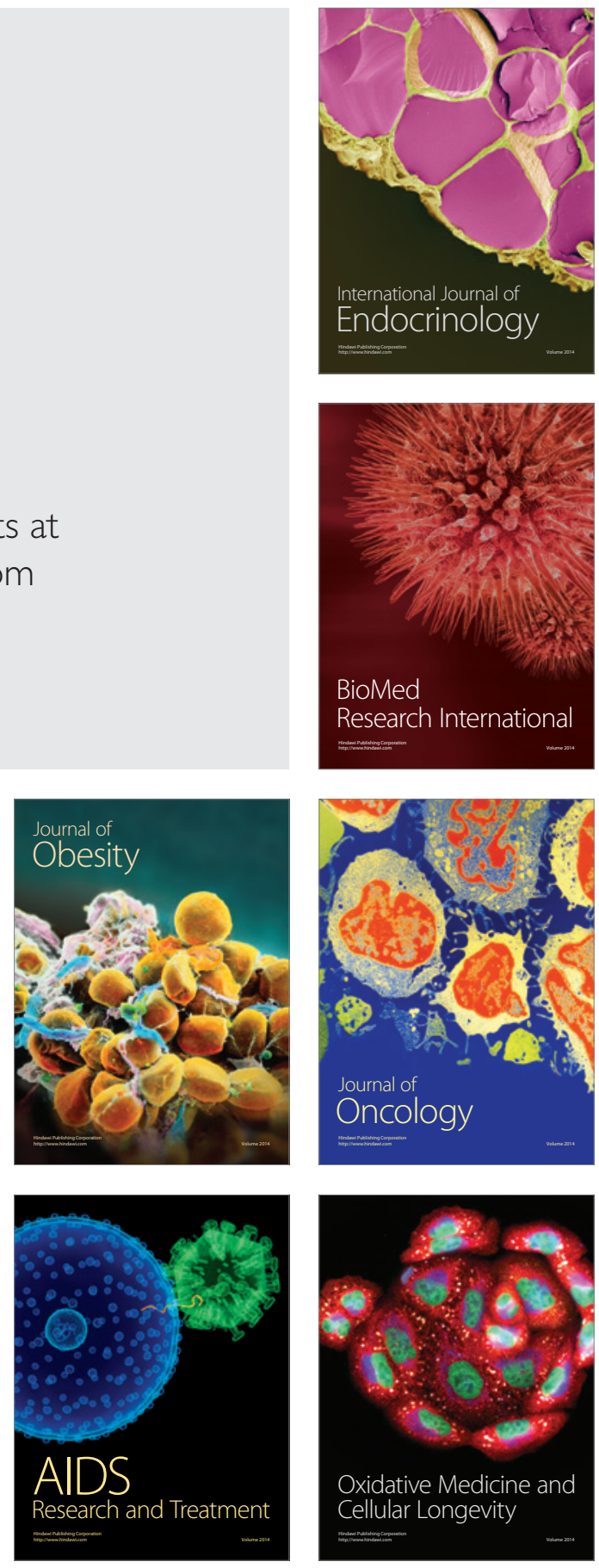